Cite this: Phys. Chem. Chem. Phys., 2014, 16, 9546

Received 13th January 2014, Accepted 31st March 2014

DOI: $10.1039 / c 4 c p 00167 b$

www.rsc.org/pccp

\section{Voltage-dependent structural changes of the membrane-bound anion channel hVDAC1 probed by SEIRA and electrochemical impedance spectroscopy $\dagger$}

\author{
Jacek Kozuch, ${ }^{* a}$ Conrad Weichbrodt, ${ }^{b}$ Diego Millo, ${ }^{c}$ Karin Giller, ${ }^{d}$ Stefan Becker, ${ }^{d}$ \\ Peter Hildebrandt ${ }^{\star a}$ and Claudia Steinem ${ }^{b}$
}

\begin{abstract}
The voltage-dependent anion channel (VDAC) is a transmembrane protein that regulates the transfer of metabolites between the cytosol and the mitochondrium. Opening and partial closing of the channel is known to be driven by the transmembrane potential via a mechanism that is not fully understood. In this work, we employed a spectroelectrochemical approach to probe the voltage-induced molecular structure changes of human VDAC1 (hVDAC1) embedded in a tethered bilayer lipid membrane on a nanostructured Au electrode. The model membrane consisted of a mixed self-assembled monolayer of 6-mercaptohexanol and (cholesterylpolyethylenoxy)thiol, followed by the deposition of 1-palmitoyl2-oleoyl-sn-glycero-3-phosphocholine vesicles including hVDAC1. The stepwise assembly of the model membrane and the incorporation of hVDAC1 were monitored by surface enhanced infrared absorption and electrochemical impedance spectroscopy. Difference spectra allowed for identifying the spectral changes which may be associated with the transition from the open to the "closed" states by shifting the potential above or below the transmembrane potential determined to be ca. $0.0 \mathrm{~V} v \mathrm{vs}$. the open circuit potential. These spectral changes were interpreted on the basis of the orientation- and distancedependent IR enhancement and indicate alterations of the inclination angle of the $\beta$-strands as crucial molecular events, reflecting an expansion or contraction of the $\beta$-barrel pore. These protein structural changes that do not confirm nor exclude the reorientation of the $\alpha$-helix are either directly induced by the electric field or a consequence of a potential-dependent repulsion or attraction of the bilayer.
\end{abstract}

\section{Introduction}

The voltage-dependent anion channel (VDAC) or mitochondrial porin plays a central role in the life and death of a cell. ${ }^{1}$ It is the most abundant protein in the mitochondrial outer membrane and governs the transport of metabolites, e.g. ATP, ADP, and glutamate, between the cytosol and the mitochondrion. ${ }^{2}$ At the same time, it was found to be an important component of a key step in apoptosis - the permeabilisation of the mitochondrial

\footnotetext{
${ }^{a}$ Technische Universität Berlin, Institut für Chemie, Straße des 17. Juni 135, D-10623 Berlin, Germany. E-mail: hildebrandt@chem.tu-berlin.de, kozuch.jacek@mailbox.tu-berlin.de

${ }^{b}$ Georg-August-Universität Göttingen, Institut für Organische und Biomolekulare Chemie, Tammannstrasse 2, 37077 Göttingen, Germany

${ }^{c}$ Vrije Universiteit Amsterdam, Biomolecular Spectroscopy/LaserLaB Amsterdam, De Boelelaan 1083, 1081 HV Amsterdam, The Netherlands

${ }^{d}$ Max-Planck Institut für Biophysikalische Chemie, Am Faßberg 11, D-37077 Göttingen, Germany

$\dagger$ Electronic supplementary information (ESI) available. See DOI: 10.1039/ c4cp00167b
}

outer membrane and the release of cytochrome $c$ from the mitochondrion into the cytosol ${ }^{3,4}$ - and thus a potential target molecule for the medication of diseases such as cancer, heart attack, Parkinson's and Alzheimer's disease. ${ }^{1}$

A particular property of the VDAC is the dependence of its channel activity on an external transmembrane potential. ${ }^{5}$ Experiments with the VDAC reconstituted into planar bilayer lipid membranes demonstrated that low potentials between ca. $-20 \mathrm{mV}$ and $20 \mathrm{mV}$ yield an open state with a conductance of ca. $4 \mathrm{nS}$ per channel in $1 \mathrm{M} \mathrm{KCl}$. However, increasing the transmembrane potential in negative and positive directions results in a "closed" conformation with a decreased conductance in the range of $c a .2 \mathrm{nS} .^{6}$ Although this effect was studied extensively, the nature of the underlying conformational changes associated with channel closing is still elusive. ${ }^{7,8}$

In 2008, three structures were published in three independent approaches, two for human VDAC1 (hVDAC1) ${ }^{9,10}$ and one for murine VDAC (mVDAC), ${ }^{11}$ and unveiled a unique structural architecture. VDAC is a $\beta$-barrel composed of an odd number of $19 \beta$-strands leading to a parallel sheet pairing of strands $\beta 1-\beta 19$. 
Furthermore, the N-terminus forms an $\alpha$-helical structure that is buried within the pore and fixed partly horizontally to the barrel wall, thus stabilizing the entire protein structure. These data were supported recently by a solid-state NMR analysis of hVDAC1 in liposomes demonstrating that the general fold of hVDAC1 is also maintained in a more native-like lipid membrane environment. ${ }^{12}$ Several models for the voltage-gating mechanism were based on rearrangements of these unique structural elements of the $\alpha$-helix and the parallel $\beta$-sheet. ${ }^{13-16}$ These theories involved, in particular, movements of the helix into the centre of the pore or realignments of the $\beta$-sheet structure to change the pore size or shape, ${ }^{13,14}$ or the electrical properties of the lumen of VDAC. ${ }^{17,18}$ Recent studies focused on the role of the $\alpha$-helix by fixing it covalently to the barrel wall. ${ }^{18,19}$ Mertins et al. ${ }^{18}$ demonstrated that fixing the helix at two different positions either impaired voltage-induced closure of the channel or produced an asymmetric gating that depended on the direction of the electric field. In contrast, Teijido et al. ${ }^{19}$ found voltageinduced gating despite restricted helix mobility. Thus, the actual role of the helix in voltage-dependent channel opening and closing is yet elusive and it remains to be clarified if, alternatively or additionally, the crucial molecular events refer to contractions and expansions of the flexible $\beta$-barrel structure.

Direct evidence for the kind of functional structure changes could be, in principle, provided by molecular spectroscopic methods, such as infrared (IR) absorption spectroscopy. ${ }^{20} \mathrm{How}^{-}$ ever, so far IR spectroscopy was only employed to determine the gross secondary structure of the protein, prior to the availability of crystallographic data. ${ }^{21,22}$ To monitor the structural changes of VDAC that are associated with the function of the channel, a spectroelectrochemical approach is required which allows monitoring the protein conformation under control of the potential. In this respect, electrode-supported bilayer lipid membranes (BLMs) represent ideal model systems as they allow embedment of the protein within the membrane and the generation of a transmembrane potential. Reconstitution of the VDAC in BLMs has in fact already been demonstrated, ${ }^{23}$ but such devices have not yet been coupled to in situ IR spectroscopic measurements. Recently, we demonstrated that the construction of a tethered bilayer lipid membrane (tBLM) on nanostructured $\mathrm{Au}$ film electrodes meets the requirements for potential-controlled IR spectroscopy since the metallic support allows for applying surface-enhanced infrared absorption (SEIRA) spectroscopy. ${ }^{24}$ Here, the nanostructured Au film is deposited on a silicon prism such that SEIRA spectroscopy can be carried out in the attenuated total reflection (ATR) mode. The Au film exerts multiple functions as the supporting material for the tBLM, the working electrode for electrochemistry, and the IR signal amplifier. Due to the distance-dependent attenuation of the signal enhancement, SEIRA allows for the detection of molecular vibrations exclusively of the tBLM and the embedded proteins. Thus, it was possible to combine electrochemical and spectroscopic measurements of the same device as documented in a recent study on the tBLMembedded peptide gramicidin $\mathrm{A}^{24}$

In the present work, we have applied this approach to hVDAC1 integrated into a tBLM system on an Au electrode.
After reconstitution of hVDAC1 into large unilamellar vesicles (LUVs), the formation of the hVDAC1-containing tBLM was monitored spectroelectrochemically using electrochemical impedance spectroscopy (EIS) and SEIRA spectroscopy. This combined approach was then used to study the electric field effects on the dynamics of the tBLM. Finally, the influence of voltage on the hVDAC1 structure was probed and the functionality was related to structural changes observed using potentialdependent SEIRA spectroscopy.

\section{Materials and methods}

\section{Materials}

6-Mercaptohexanol $(6 \mathrm{MH})$ and $N$-lauryl- $N, N$-dimethylamine- $N$ oxide (LDAO) were purchased from Sigma Aldrich. 1-Palmitoyl2-oleoyl-sn-glycero-3-phosphocholine (POPC) and Bio-Beads SM2 were obtained from Avanti Polar Lipids and Bio-Rad, respectively. (Cholesterylpolyethylenoxy)thiol (CPEO3) was synthesized according to Schmitt et al. ${ }^{25}$ Other chemicals were of highest purity grade available. All solutions were prepared using MilliQ water $(>18 \mathrm{M} \Omega \mathrm{cm}) \cdot \mathrm{D}_{2} \mathrm{O}$ and $\mathrm{DCl}$ were purchased from Deutero GmbH.

\section{Protein expression and purification}

hVDAC1 was overexpressed in Escherichia coli, purified and refolded into micelles consisting of the detergent $N$-lauryl- $N, N$-dimethylamine- $N$-oxide (LDAO) as described previously. ${ }^{22}$

\section{Vesicle preparation}

Unilamellar POPC vesicles with a final concentration of $0.5 \mathrm{mg} \mathrm{mL}^{-1}$ were prepared by the extrusion method according to Schmitt et $a .^{25}$ using polycarbonate filters with a nominal size of $100 \mathrm{~nm}$ and a $20 \mathrm{mM}$ Tris-HCl buffer solution including $100 \mathrm{mM} \mathrm{NaCl}$, adjusted to $\mathrm{pH} 7.4$ (pD 7.8) by $\mathrm{HCl}$ (DCl). For hVDAC1 incorporation, unilamellar vesicles were prepared and mixed with LDAO to final concentrations of $0.5 \mathrm{mg} \mathrm{mL}^{-1}$ POPC and 6.5 mM LDAO. hVDAC1 was incubated at a final concentration of $1 \mu \mathrm{M}$ with the vesicle-detergent mixture for $30 \mathrm{~min}$ at $20{ }^{\circ} \mathrm{C}$. The detergent was removed using $2 \times 25 \mathrm{mg}$ BioBeads SM2 overnight at $4{ }^{\circ} \mathrm{C}$.

\section{tBLM assembly}

A nanostructured Au film was formed on the silicon prism by electroless deposition. ${ }^{26}$ The mixed CPEO3/6MH SAM was selfassembled on the Au film overnight from a solution of $0.05 \mathrm{mM}$ CPEO3 and $0.05 \mathrm{mM} 6 \mathrm{MH}$ in isopropanol yielding a phaseseparated surface composition of $80 \%$ CPEO3 and $20 \% 6 \mathrm{MH}$ as determined from the capacitance of $1.4 \mu \mathrm{F} \mathrm{cm} \mathrm{cm}^{-2}$ (capacitances of pure CPEO3 and $6 \mathrm{MH}$ on the Au film were determined to be $(1.02 \pm 0.06)$ and $(3.09 \pm 0.11) \mu \mathrm{F} \mathrm{cm}^{-2}$, respectively). ${ }^{27}$ Pure or hVDAC1-containing unilamellar POPC vesicles were added on the SAM to form the tBLM. After 3 hours, incubation was aborted by exchange with buffer solution. To improve the quality of the tBLM, an electrochemical treatment (ETC) was 
performed by switching between the open circuit potential and a $60 \mathrm{mV}$ higher potential 20 times at $5 \mathrm{~min}$ intervals.

\section{Electrochemical impedance and surface enhance infrared absorption spectroscopy}

Spectroelectrochemical experiments were performed using a nanostructured Au film formed on a trapezium-shaped silicon ATR-IR element $\left(W \times L \times H=20 \times 25 \times 10 \mathrm{~mm}^{3}\right)$. All electrochemical experiments were recorded using a $\mu$ AutolabIII/ FRA2. EIS was controlled using the FRA software and performed using the Au film as the working electrode with a real surface area of $1.65 \mathrm{~cm}^{2}$ (determined by the Au-oxide reduction charge density method; geometric area of $0.79 \mathrm{~cm}^{2}$ with a roughness factor of 2.1). A Pt-wire and an $\mathrm{Ag} / \mathrm{AgCl}$ electrode $(3 \mathrm{M} \mathrm{KCl}$ ) served as counter and reference electrodes, respectively. Spectra were recorded in the frequency range of $0.05 \mathrm{~Hz}$ to $100 \mathrm{kHz}$ at a potential of $0 \mathrm{~V}$ ( $v s$. $\mathrm{Ag} / \mathrm{AgCl})$ and an amplitude of $25 \mathrm{mV}(\mathrm{rms})$.

SEIRA spectroscopy was performed using a Bruker IFS66v/s spectrometer equipped with a photoconductive MCT detector and a Kretschmann-ATR setup under an angle of incidence of $60^{\circ}$. Each spectrum consisted of 400 scans and was recorded in a spectral window of 4000 to $1000 \mathrm{~cm}^{-1}$ with a resolution of $4 \mathrm{~cm}^{-1}$. Potential-dependent difference spectra were obtained by measuring spectra of two respective potentials (e.g. A and B) in the sequence $\mathrm{A} \rightarrow \mathrm{B} \rightarrow \mathrm{B} \rightarrow \mathrm{A}$. Thus, possible long-term effects were efficiently reduced in the "B-minus-A" difference spectrum. To increase the signal-to-noise ratio, this sequence was repeated 40 times.

\section{Results and discussion}

\section{Electrochemical impedance spectroscopy}

Fig. 1 shows the EIS data, represented in the Cole-Cole plot, for the mixed SAM and the tBLM systems with and without hVDAC1. As already demonstrated in our previous work, ${ }^{24}$ the nanostructured Au surface, needed for the surface-enhancement of the IR signals, affords two dispersions in the EIS data. This can be rationalized on the basis of an extended aqueous reservoir in the regions of pure CPEO3 islands, separating the capacitive behaviour of the spacer region from the cholesteryl headgroup or bilayer region. In the Cole-Cole plot (Fig. 1, left), the first half circle at high frequencies describes the capacitive behaviour of the entire SAM or tBLM. Thus, to exclude the effect of a model-dependent fit to the EIS data, we determine the value of the capacitance directly by reading out the diameter of the half-circle. ${ }^{27}$ Upon addition of pure and hVDAC1-containing vesicles, the capacitance of the mixed SAM decreases from $1.4 \mu \mathrm{F} \mathrm{cm} \mathrm{cm}^{-2}$ to $c a$. $0.64 \mu \mathrm{F} \mathrm{cm} \mathrm{cm}^{-2}$. This value is in line with literature data for lipid membranes ${ }^{28}$ and, hence, proves the formation of the tBLM also in the presence of hVDAC1. The tBLM resistance can be estimated using the equivalent circuit $R_{\text {Solution }}\left(R_{\text {Spacer }} C_{\text {Spacer }}\right)\left(R_{\text {Bilayer }} C_{\text {Bilayer }}\right) Q$ accounting for the resistance and the capacitance of the two dispersions of the electrode-facing spacer and the solution-exposed bilayer region (see ESI, $\dagger$ Fig. S1). ${ }^{24}$ Although the "tails" of the impedance data at low frequencies indicate that the tBLM is not an ideal capacitor, we have chosen capacitor elements over constant-phase elements for the fit due to their intuitive physical meaning. To account for the "tails" a constant-phase element (CPE) $Q$ was introduced. This CPE describes the nonideal electrical behaviour of the system which is based on a combination of the flexibility of the CPEO3 SAM and tBLM or the interfacial polarisation known as the Maxwell-WagnerSiller effect. ${ }^{27,29}$ The results of fitting this equivalent circuit to the experimental data are listed in Table 1. The resistance of the bilayer $R_{\text {Bilayer }}$ shows nearly the same value of $c a .1 .1$ and $1.4 \mathrm{k} \Omega \mathrm{cm}^{2}$ in the presence and absence of hVDAC1, respectively. The lack of a significant difference between hVDAC1tBLMs and pure tBLMs is attributed to the presence of defects that, before the ECT, exhibit a higher conductivity than the ion channel, and to an unfavourable orientation and positioning of hVDAC1 molecules that impairs ion transfer. This is the case for proteins anchored within the lipid monolayer on top of the
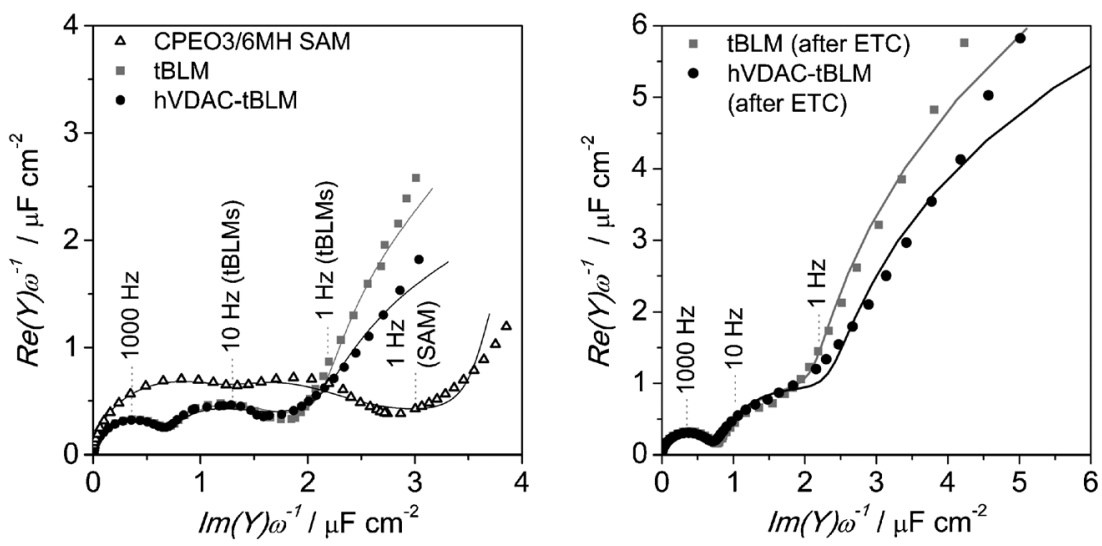

Fig. 1 Cole-Cole representations of the EIS data of the formation of tBLMs on the nanostructured Au electrode before (left) and after (right) the ETC. Lines represent the fit of the equivalent circuit $R_{\text {Solution }}\left(R_{\text {Bilayer }} C_{\text {Bilayer }}\right)\left(R_{\text {spacer }} C_{\text {spacer }}\right) Q$ (see ESI $\left.\dagger\right)$ to the respective spectra. Hollow triangles refer to the impedance of the mixed CPEO3/6MH SAM; grey squares and black circles represent the tBLM and hVDAC1-tBLM, respectively. Labels indicate several frequencies of the EIS data. 
Table 1 Results of fitting the equivalent circuit $R_{\text {Solution }}\left(R_{\text {Bilayer }} C_{\text {Bilayer }}\right)\left(R_{\text {Spacer }} C_{\text {Spacer }}\right) Q$ to the EIS data (see Fig. 1) $)^{a}$

\begin{tabular}{|c|c|c|c|c|c|}
\hline & СРEO3/6MH SAM & tBLM & hVDAC1-tBLM & tBLM (after ETC) & hVDAC1-tBLM (after ETC) \\
\hline$C_{\mathrm{tBLM}}{ }^{a}\left[\mu \mathrm{F} \mathrm{cm}^{-2}\right]$ & 1.4 & 0.63 & 0.64 & 0.66 & 0.61 \\
\hline$R_{\text {Bilayer }}\left[\mathrm{k} \Omega \mathrm{cm}^{2}\right]$ & 0.2 & 1.4 & 1.1 & 19.9 & 8.8 \\
\hline$C_{\text {Bilayer }}\left[\mu \mathrm{F} \mathrm{cm}^{-2}\right]$ & 7.40 & 2.18 & 2.86 & 1.67 & 1.83 \\
\hline$C_{\text {Spacer }}\left[\mu \mathrm{F} \mathrm{cm}^{-2}\right]$ & 4.94 & 3.39 & 5.65 & 3.20 & 4.47 \\
\hline$Q\left[\mu \mathrm{F} \mathrm{s}^{\alpha-1}\right]$ & 15.9 & 8.5 & 5.9 & 14.7 & 12.71 \\
\hline$\alpha$ & 0.79 & 0.81 & 0.82 & 0.77 & 0.76 \\
\hline
\end{tabular}

${ }^{a}$ The capacitance value of the tBLM $\left(C_{\mathrm{tBLM}}\right)$ was determined directly from the Cole-Cole plot as described in the text. Errors (standard deviation between experiments) of the resistances are $10 \%$ whereas those of the capacitances and the $Q$ value are $5 \%$. $(n=3)$.

CPEO3 monolayer instead of being integrated in the complete bilayer (vide infra).

Applying the ECT leads to a significant increase of the quality of the tBLM systems (Fig. 1, right). The first half circle becomes more pronounced with only minor changes in the capacitance and the second half circle diminishes. The resistance $R_{\text {Bilayer }}$ in the absence and the presence of hVDAC1 increases to $19.9 \mathrm{k} \Omega \mathrm{cm}^{2}$ and $8.8 \mathrm{k} \Omega \mathrm{cm}^{2}$, respectively. This difference can be readily understood, since the hVDAC1 pore allows for a diffusion of ions across the membrane, which decreases the membrane resistance. We further conclude that the ECT does not only reduce the number of defects, but might also shift the protein into a more favourable position in the $6 \mathrm{MH}$-supported bilayer fragments of the tBLM (vide infra).

\section{SEIRA spectroscopy}

Fig. 2 shows SEIRA difference spectra of the formation of both tBLM systems before (left) and after the ECT (right) taking the spectrum of the mixed SAM as the reference. In this way, positive and negative IR bands are attributed to species being adsorbed and removed from the Au surface, respectively. These spectra display the typical features of tBLM formation. ${ }^{24}$ In the spectra obtained from samples in $\mathrm{H}_{2} \mathrm{O}$, the broad negative band centred at ca. $1645 \mathrm{~cm}^{-1}$ (ascribed to the $\mathrm{H}_{2} \mathrm{O}$ bending mode)
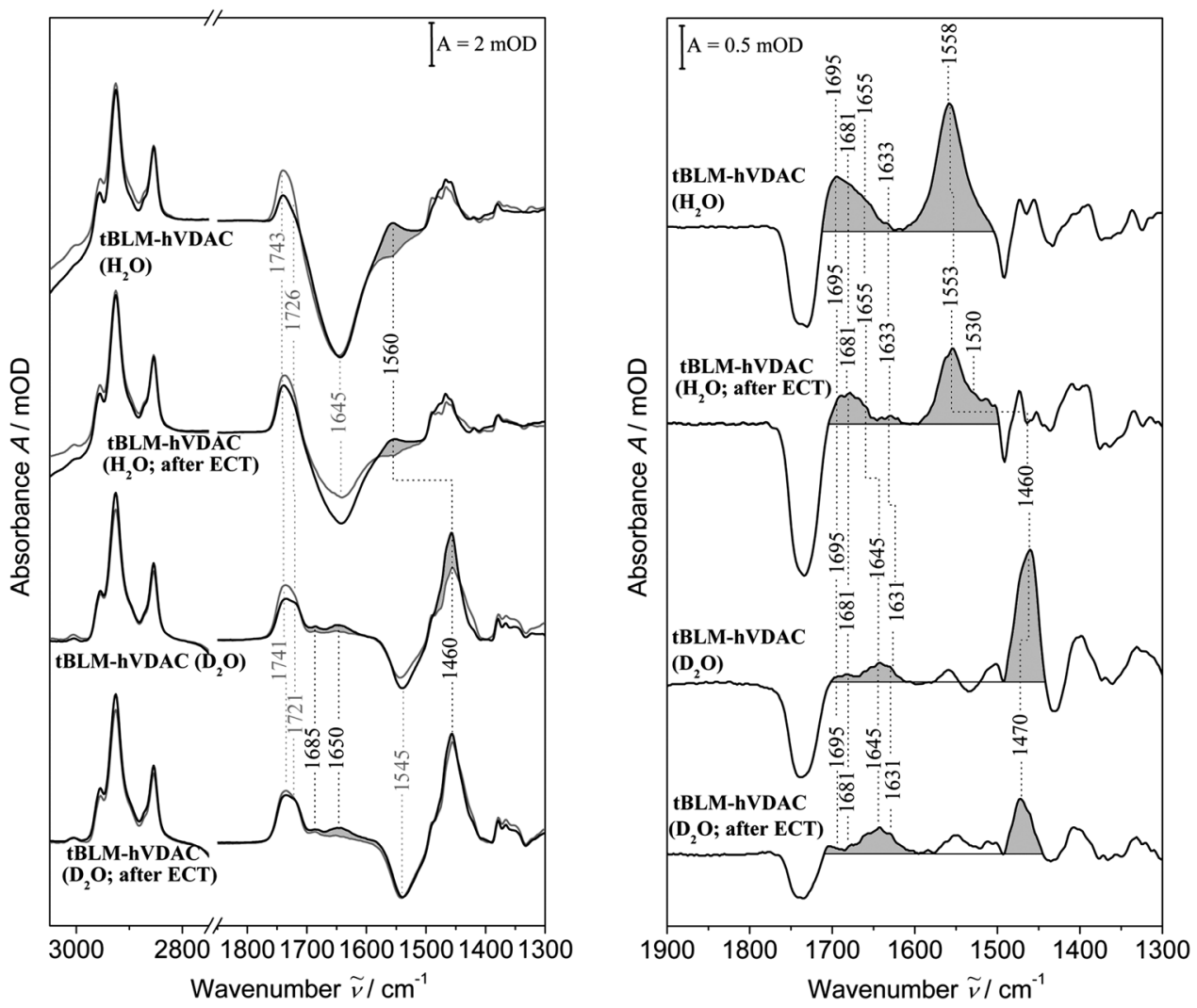

Fig. 2 Left: SEIRA difference spectra of tBLMs (grey) and hVDAC1-tBLMs (black) recorded for samples in $\mathrm{H}_{2} \mathrm{O}$ and $\mathrm{D}_{2} \mathrm{O}$, using the spectrum of the mixed SAM as the reference spectrum. Black numbers refer to bands of hVDAC1 and grey numbers to lipid and water. Right: SEIRA difference spectra calculated by subtracting the pure tBLM spectrum from the respective spectra of hVDAC1-tBLM on the left side. In both panels, amide I and II bands are marked in grey. 
reflects the removal of water from the SAM-coated electrode due to spreading of POPC vesicles on the mixed SAM (Fig. 2, left). The process of tBLM formation can be monitored by the positive bands between $3050-2800 \mathrm{~cm}^{-1}$ and at $c a .1730 \mathrm{~cm}^{-1}$ which are attributed to the $\mathrm{CH}_{n}(n=1,2,3)$ and the $\mathrm{C}=\mathrm{O}$ stretching modes of the acyl chains of POPC molecules, respectively. When this experiment is performed in $\mathrm{D}_{2} \mathrm{O}$, the broad negative bending mode of $\mathrm{D}_{2} \mathrm{O}$ is located at $c a .1210 \mathrm{~cm}^{-1}$ (not shown here) and the $\mathrm{C}=\mathrm{O}$ stretching shows a slight downshift compared to the spectrum of the sample in $\mathrm{H}_{2} \mathrm{O}$. The $\mathrm{C}=\mathrm{O}$ stretching mode is composed of two components located at 1743 and $1726 \mathrm{~cm}^{-1}$ and attributable to the carboxyl functions in non-hydrogen-bond (or weak-hydrogen-bond) and hydrogenbond environments, respectively. In the presence of $\mathrm{D}_{2} \mathrm{O}$, in particular the low-frequency component of the hydrogenbonded species displays a downshift to $1721 \mathrm{~cm}^{-1}$; the highfrequency $\mathrm{C}=\mathrm{O}$ stretching shifts only slightly to $1741 \mathrm{~cm}^{-1}$. Furthermore, the SEIRA difference spectra are dominated by a negative and a positive band at 1545 and $1460 \mathrm{~cm}^{-1}$, respectively, which arise from the H/D-exchange at the carbamate $\mathrm{N}-\mathrm{H}$ group of the CPEO3 molecules. Since the negative band at $1645 \mathrm{~cm}^{-1}\left(\mathrm{H}_{2} \mathrm{O}\right.$ bending mode) interferes with the amide I band, which, in contrast to the amide II band, is only weakly $\mathrm{H} / \mathrm{D}$-sensitive, information about protein structural changes was primarily extracted from the analysis of amide $\mathrm{I}^{\prime}$ (the prime denotes the amide band in $\mathrm{D}_{2} \mathrm{O}$ ) and amide II bands in the spectra obtained from samples in $\mathrm{D}_{2} \mathrm{O}$ and $\mathrm{H}_{2} \mathrm{O}$, respectively.

Prior to the ECT, the $\mathrm{CH}_{n}$ stretching region is very similar in the spectra of the hVDAC1-containing and the pure tBLMs, both in the case of $\mathrm{H}_{2} \mathrm{O}$ and $\mathrm{D}_{2} \mathrm{O}$. This finding indicates that the presence of hVDAC1 within the vesicles promotes the formation of tBLMs having a similar overall bilayer structure as in the case of pure tBLMs. This is in line with the EIS results showing the typical dielectric properties of a tBLM for both systems (vide supra). However, the $\mathrm{C}=\mathrm{O}$ stretching reveals different intensities and slightly altered band shapes. This difference may be attributed to a modified arrangement of the $\mathrm{C}=\mathrm{O}$ bonds of the POPC molecules caused by defects within the tBLM, as already suggested by the EIS data. Upon ECT, this difference is diminished and these bands approach intensities similar to those of the pure tBLMs, concomitant with an increase in membrane resistance that reflects the improvement of the order of the hVDAC1-tBLM system, and thus of its insulating properties. The yet remaining intensity difference of the $\mathrm{C}=\mathrm{O}$ signal can then be attributed to the lower number of POPC molecules in the presence of hVDAC1 and an altered $\mathrm{C}=\mathrm{O}$ orientation within the tBLM due to specific protein-lipid interactions.

The most prominent feature in the spectra of the hVDAC1containing tBLMs is the amide II band of the protein at $1560 \mathrm{~cm}^{-1}$ in $\mathrm{H}_{2} \mathrm{O}$. This IR band which is missing in the spectra of pure tBLMs proves the incorporation of hVDAC1 into the tBLM. The corresponding amide I modes appear between 1700 and $1630 \mathrm{~cm}^{-1}$ and are obscured by the intense negative water band at ca. $1645 \mathrm{~cm}^{-1}$ (vide supra). Nevertheless, one may analyze this region on the basis of the difference spectrum that was obtained by subtracting the spectrum of the pure tBLM from that of the hVDAC1-tBLM such that the negative water band vanished (Fig. 2, right). This spectrum reveals the amide I components albeit with somewhat distorted band shapes due to the subtraction procedure. Four components at 1695, 1681, 1655 and $1633 \mathrm{~cm}^{-1}$ can be identified and ascribed to the minor $\beta$-sheet, turns, random coils, and the major $\beta$-sheet band of hVDAC1, respectively. ${ }^{30}$ The $1655 \mathrm{~cm}^{-1}$ band might also include contributions from the $\alpha$-helix. However, since this structural element is preferentially oriented parallel to the membrane and thus also to the Au surface, the transition dipole moment of its amide I mode adopts the same parallel orientation such that this mode experiences no surface enhancement and its contribution to the $1655 \mathrm{~cm}^{-1}$ band should be rather weak. ${ }^{31}$ The amide II has a maximum at $1558 \mathrm{~cm}^{-1}$ with a small shoulder at $c a .1530 \mathrm{~cm}^{-1}$. At first glance, this amide II band pattern seems to be in contradiction to the previous ATR-IR spectra of hVDAC1 that show a broad amide II band centred at $1530 \mathrm{~cm}^{-1} \cdot{ }^{21}$ In the case of SEIRA spectroscopy, however, the preferential enhancement for structural elements in close proximity to the Au surface has to be taken into account. These are mainly $\beta$-sheet-connecting turns and random coils. The amide II modes of these secondary structures are in fact observed at $c a .1560 \mathrm{~cm}^{-1},,^{20,32}$ and thus are likely to be the main origin of the SEIRA band at $1558 \mathrm{~cm}^{-1}$. Additional contributions may come from the amide modes of asparagine and glutamine side chains. ${ }^{20}$ The $\alpha$-helix does not contribute (or only weakly) to the amide II band, since it is expected at $c a .1545 \mathrm{~cm}^{-1} .^{32}$

In order to resolve the amide I region more clearly, SEIRA spectra of pure tBLMs and hVDAC1-tBLMs were measured from samples in $\mathrm{D}_{2} \mathrm{O}$ (Fig. 2, right). After applying the subtraction procedure in the same way as in the case of $\mathrm{H}_{2} \mathrm{O}$, the difference spectrum displays the amide $\mathrm{I}^{\prime}$ region with the partly downshifted band components at 1695 (minor $\beta$-sheet), 1681 (turns), 1645 (random coils), and $1631 \mathrm{~cm}^{-1}$ (major $\beta$-sheet). Here, the amide $\mathrm{I}^{\prime}$ region is in good agreement with literature data. ${ }^{21}$ The $\beta$-sheet absorption at $1631 \mathrm{~cm}^{-1}$ is more pronounced than its counterpart in $\mathrm{H}_{2} \mathrm{O}$, but still weaker than the band at $1645 \mathrm{~cm}^{-1}$ which can be rationalized by taking into account the surface-selection rules and the distance-dependent attenuation of the enhancement. The more abundant $\beta$-sheets $\left(1631 \mathrm{~cm}^{-1}\right)$ are farther away from the Au surface than the random coil motifs and turns, both of which are located at the entrance of the ion channel. Correspondingly, we note a weaker band at $1530 \mathrm{~cm}^{-1}$ ( $\beta$-sheet) and a more intense band at the $1558 \mathrm{~cm}^{-1}$ amide II band in $\mathrm{H}_{2} \mathrm{O}$ (turns and random coil).

The overall intensity of the amide $\mathrm{I}\left(\mathrm{H}_{2} \mathrm{O}\right)$ and amide $\mathrm{I}^{\prime}$ bands $\left(\mathrm{D}_{2} \mathrm{O}\right)$ of $c a$. $0.3 \mathrm{mOD}$ points to a low protein density in the tBLM. In comparison, protein monolayers studied using SEIRA spectroscopy yielded intensities that were higher by a factor of 10 to $50 .^{33}$ One may estimate a degree of protein incorporation into the tBLM that corresponds to $\mathrm{ca}$. $1-10 \%$ of the geometrically possible maximum value. This value is consistent with an estimate derived from the specific area required for POPC $\left(68 \AA^{2}\right)^{34}$ and hVDAC1 molecules $\left(852 \AA^{2}\right)^{10}$ and the 
molar lipid/protein ratio of 660:1 in the initial hVDAC1containing vesicles that leads to a surface occupancy of hVDAC1 of $c a \cdot 3.7 \%$. Thus, a mixed SAM composed of $80 \%$ of CPEO3 provides sufficient space for all hVDAC1 molecules to occupy the $6 \mathrm{MH}$-supported bilayer fragments.

After the ECT, substantial changes are observed in the amide region of the protein. First of all, the amide I to amide II ratio

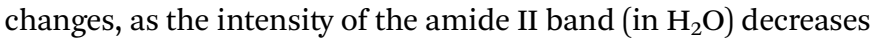
and the amide $\mathrm{I}^{\prime}$ (in $\mathrm{D}_{2} \mathrm{O}$ ) increases slightly. Furthermore, the amide II shifts from 1558 to $1553 \mathrm{~cm}^{-1}$ and the shoulder at $1530 \mathrm{~cm}^{-1}$ becomes more pronounced. This observation points at a rearrangement of the entire protein towards a more uniform orientation within the tBLM. This is in line with the results from the impedance spectra, indicating a lower resistance of the hVDAC1-containing tBLM after the ECT compared to the pure tBLM. The shift of the amide $\mathrm{II}^{\prime}$ band from 1460 to $1470 \mathrm{~cm}^{-1}$ might be associated with this reorientation as well, but since it is superimposed by the N-D stretching of the CPEO3 molecules and modes of amino acid residues, a more specific interpretation is not possible.

\section{Electric field effects on the tBLM}

To investigate the structural changes associated with the functionality of hVDAC1, potential-dependent SEIRA difference spectra of hVDAC1-containing tBLMs were recorded. However, these spectra are strongly affected by electric field-driven changes in the tBLM itself. These effects are already observable in the low frequency "tail" of the impedance spectra that indicate a deviation of the tBLM from the behaviour of an ideal capacitance towards a frequency-dependent capacitance which we ascribe to the high flexibility of the CPEO3 SAM and the
tBLM as a whole. Thus, for a better understanding of the structural changes observed in the difference spectra, first the pure tBLM system will be treated.

In a previous study, Jeuken and coworkers ${ }^{35}$ studied the electric-field effects on the tBLM of the same architecture using a combined electrochemical and atomic force microscopic (AFM) approach. In that study, global structural changes of the bilayer were observed in which the $6 \mathrm{MH}$-supported bilayer was elevated with respect to the electrode surface at $c a .-450 \mathrm{mV}$ (vs. $\mathrm{Ag} / \mathrm{AgCl}$ ).

In the present work, similar movements of the bilayer can be derived from the potential-dependent changes of the SEIRA spectrum. Fig. 3 (left) presents the SEIRA difference spectra obtained from spectra measured at different electrode potentials. These difference spectra were obtained over a wide potential window keeping always a constant potential difference of $60 \mathrm{mV}$ between the applied voltages. The results show that the difference spectra "+60 $\mathrm{mV}$ minus $0 \mathrm{mV}$ " and "-115 $\mathrm{mV}$ minus $-175 \mathrm{mV}$ " (vs. $\mathrm{Ag} / \mathrm{AgCl}$ ) are very similar with only slightly different relative intensities. First, the negative band of the $\mathrm{H}_{2} \mathrm{O}$ stretching at $c a .3400 \mathrm{~cm}^{-1}$ indicates the removal of water from the vicinity of the Au surface. Furthermore, two prominent negative bands are observed at 1698 and $1562 \mathrm{~cm}^{-1}$ corresponding to the $\mathrm{C}=\mathrm{O}$ stretching and the $\mathrm{N}-\mathrm{H}$ bending of CPEO3 (Fig. 3, top spectrum). These difference bands are very intense since the CPEO3 molecule covers $80 \%$ of the $\mathrm{Au}$ surface and thus also strongly benefit from the surfaceenhancement. The positive band at $1272 \mathrm{~cm}^{-1}$ originates from a superposition of bands of various tBLM components, in particular the $\mathrm{C}-\mathrm{N}$ stretching of CPEO3 and the $\mathrm{O}-\mathrm{H}$ bending of $6 \mathrm{MH}$, but also the asymmetric $\mathrm{PO}_{2}$ stretching $\left(\mathrm{ca} .1235 \mathrm{~cm}^{-1}\right)$
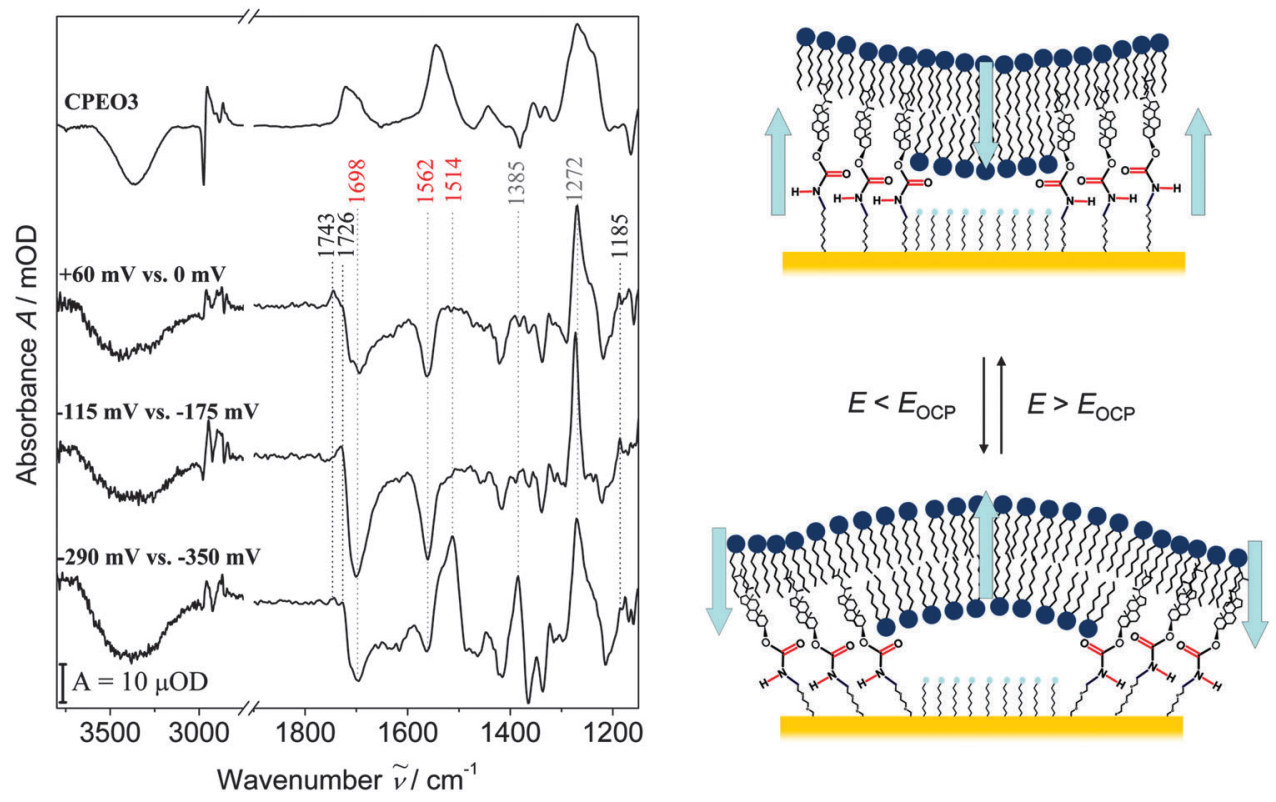

Fig. 3 Left: potential-dependent SEIRA difference spectra of pure tBLMs recorded between the potentials of +60 and $0 \mathrm{mV}$, -115 and $-175 \mathrm{mV}$, and -290 and $-350 \mathrm{mV}$ (vs. Ag/AgCl). The SEIRA spectrum of a CPEO3 SAM, recorded vs. 1-propanol, is given on the top for comparison. Bands of POPC and CPEO3 are labeled in black and red, respectively. Right: schematic model for the electric field-dependent effects on the tBLM. Red bonds of the CPEO3 molecules refer to the red labels in the spectra on the left side. 
and the choline C-N stretching ( $c a .1260 \mathrm{~cm}^{-1}$ ) of the headgroups of POPC (see ESI $\dagger$ ) and, thus, is more difficult to interpret. The $\mathrm{C}=\mathrm{O}$ stretching $\left(\mathrm{ca} .1730 \mathrm{~cm}^{-1}\right)$ and the asymmetric $\mathrm{C}-\mathrm{O}-\mathrm{C}$ stretching $\left(1185 \mathrm{~cm}^{-1}\right)$ of the POPC molecules show positive bands with lower intensity since these groups are located much farther away from the electrode surface. These spectral features can be interpreted in terms of collective movements of the tBLM brought about by the action of the electric field on the membrane (Fig. 3, right): upon application of more positive potentials, the bilayer segments located on top of the 6MH SAM are pulled towards the Au surface, accompanied by the release of water from the sub-membrane reservoir. At the same time, the CPEO3 molecules are tilted up, so the distance between the carbamate group and the Au surface is increased. Reorientation of the CPEO3 molecules can be excluded on the basis of the transition dipole moments of the $\mathrm{C}=\mathrm{O}$ stretching and the $\mathrm{N}-\mathrm{H}$ bending that are oriented nearly perpendicularly with respect to each other. This movement probably compensates the bending of the bilayer and minimizes the friction within the tBLM.

The SEIRA difference spectrum "-290 mV vs. $-350 \mathrm{mV}$ " (vs. $\mathrm{Ag} / \mathrm{AgCl}$ ) differs considerably from the other spectra since new positive bands dominate the spectrum. These are the symmetric $\mathrm{CH}_{3}$ deformation at $1385 \mathrm{~cm}^{-1}$ of either CPEO3 or POPC, ${ }^{36}$ and the prominent band at $1514 \mathrm{~cm}^{-1}$ that is assigned to the weakly hydrogen-bonded $\mathrm{N}-\mathrm{H}$ group (see ESI $\dagger$ ). The changes in this difference spectrum can be interpreted in terms of a reorientation of the CPEO3 molecules. Accordingly, at more positive potentials the $\mathrm{CPEO} 3$ molecules are inclined towards the surface normal leading to a more parallel orientation of the $\mathrm{C}=\mathrm{O}$ and a more perpendicular orientation of the $\mathrm{N}-\mathrm{H}$ transition dipole moments with respect to the Au surface, corresponding to a lower enhancement of the $\mathrm{C}=\mathrm{O}$ stretching at $1698 \mathrm{~cm}^{-1}$ and an increased enhancement of the $\mathrm{N}-\mathrm{H}$ bending at $1514 \mathrm{~cm}^{-1}$. Conversely, at more negative potentials the CPEO3 molecules are inclined towards the $\mathrm{Au}$ surface. This orientation may also cause the weakening of the $\mathrm{H}$-bond of the $\mathrm{N}-\mathrm{H}$ group. The still persisting negative $\mathrm{N}-\mathrm{H}$ band at $1562 \mathrm{~cm}^{-1}$ reflects the movement of $\mathrm{H}$-bonded CPEO3 molecules in the vicinity of the $6 \mathrm{MH}$-coated areas. The disappearance of the positive signals at 1743 or $1726 \mathrm{~cm}^{-1}$ can be tentatively explained in terms of a rearrangement of the $\mathrm{C}=\mathrm{O}$ groups that brings these groups into an orientation of lower surface enhancement or stronger hydrogen bond interactions. As a consequence, the $\mathrm{C}=\mathrm{O}$ stretching is obscured by the adjacent strong negative signal. Besides this, the pattern of negative bands is quite similar to that in the previous spectra. Consequently, we conclude that the SEIRA difference spectrum "-290 $\mathrm{mV}$ vs. $-350 \mathrm{mV}$ " (vs. Ag/AgCl) reflects the transition from the perturbed tBLM, where the bilayer is pulled towards the surface, to a "relaxed" state. The potential that characterizes this transition thus lies in the range between $-290 \mathrm{mV} v s$. $-350 \mathrm{mV}$ (vs. $\mathrm{Ag} / \mathrm{AgCl})$ and is close to the potential at open circuit $\left(E_{\mathrm{OCP}}\right)$ which was determined to be at $c a .-350 \mathrm{mV}$ vs. $\mathrm{Ag} / \mathrm{AgCl}$. In a previous study on $\mathrm{tBLM}, E_{\mathrm{OCP}}$, i.e. the potential of zero current, was found to be similar to the potential of zero charge $\left(E_{\mathrm{PZC}}\right)$ albeit with a different value for $E_{\mathrm{PZC}} \approx E_{\mathrm{OCP}}{ }^{35}$ Attributing this numerical difference to the different lipid composition of the tBLM and the defect-induced decreased crystalline character of the $6 \mathrm{MH}$ SAM fragments, we assume that the relationship $E_{\mathrm{PZC}} \approx E_{\mathrm{OCP}}$ also holds for the tBLM used in this work. Then the SEIRA spectroscopic changes discussed above can readily be rationalized since crossing the transition potential $\left(\approx E_{\mathrm{PZC}}\right)$ results in qualitatively different electrostatic interactions due to the reversal of the interfacial electric field vector. As a consequence the attractive forces which cause the deformation of the bilayer at $E<E_{\mathrm{PzC}}$ are steadily released when the potential approaches $E_{\mathrm{PZC}}$.

\section{Voltage-induced structural changes of hVDAC1}

It was previously reported that tBLMs with a resistance in the order of $\mathrm{M} \Omega \mathrm{cm}^{2}$ can be used to monitor currents from single channel opening and closing events. ${ }^{37,38}$ Unfortunately, in the present tBLM system, potential-dependent currents did not allow for direct monitoring of the transition between "open" and "closed" states of an ensemble of hVDAC1 (see ESI, $\dagger$ Fig. S6 and S7). Thus, we tried to detect "closing" and "opening" of hVDAC1 integrated in tBLMs by SEIRA difference spectroscopy. In experiments using freestanding BLMs with two equivalent electrodes, the open state is observed at a potential that is defined by the zero-current (see ESI, $\dagger$ Fig. S2-S4), which for the tBLM device corresponds to $E_{\mathrm{OCP}}$. Therefore, the SEIRA spectrum recorded at $E_{\mathrm{OCP}}$ $(-350 \mathrm{mV}$ vs. $\mathrm{Ag} / \mathrm{AgCl})$ was subtracted from those measured at $E_{\mathrm{OCP}}+60 \mathrm{mV}$ and $E_{\mathrm{OCP}}-60 \mathrm{mV}$, yielding "closed-minus-open" difference spectra. Since the potential-dependent changes of the bilayer discussed in the previous section superimpose the changes of the protein, the same difference spectra were recorded for pure tBLMs. Fig. 4 shows the difference spectra for hVDAC1tBLMs and pure tBLMs. In both cases, the spectra show mirrorlike features for positive and negative potentials, and thus, in the following, the " $E_{\mathrm{OCP}}+60 \mathrm{mV}$ " vs. " $E_{\mathrm{OCP}}$ " difference spectra will be discussed unless noted otherwise.

Upon incorporation of hVDAC1, several changes were observed in the spectra. First, the negative band at $c a .3400 \mathrm{~cm}^{-1}$, reflecting the depletion of water from the sub-membrane region, shows a lower intensity in the presence of hVDAC1. This may be attributed to the extra water molecules that are accommodated in the pore of hVDAC1 and thus contribute to the SEIRA signal. Therefore, less membrane material (i.e. volume of molecules other than water) is relocated upon application of potentials, resulting in a smaller volume of water that is removed or added concomitantly. Consequently, the intensity of the difference band is lower.

The most important spectral changes refer to the region of the amide I and amide II modes between 1700 and $1500 \mathrm{~cm}^{-1}$. Since $\mathrm{CPEO} 3$ also contains $\mathrm{C}=\mathrm{O}$ stretching and $\mathrm{N}-\mathrm{H}$ bending modes, the respective difference bands may overlap with the changes of the amide I and amide II bands of hVDAC1. To sort out the structural changes of the protein and the membrane, SEIRA double-difference spectra were constructed by subtracting the difference spectra of pure tBLMs from those of hVDAC1tBLMs without any further correction neither of the intensity of the spectra nor of the baseline (Fig. 4). Thus, these SEIRA double-difference spectra display solely changes attributable to hVDAC1 and protein-membrane interactions. The amide I region 

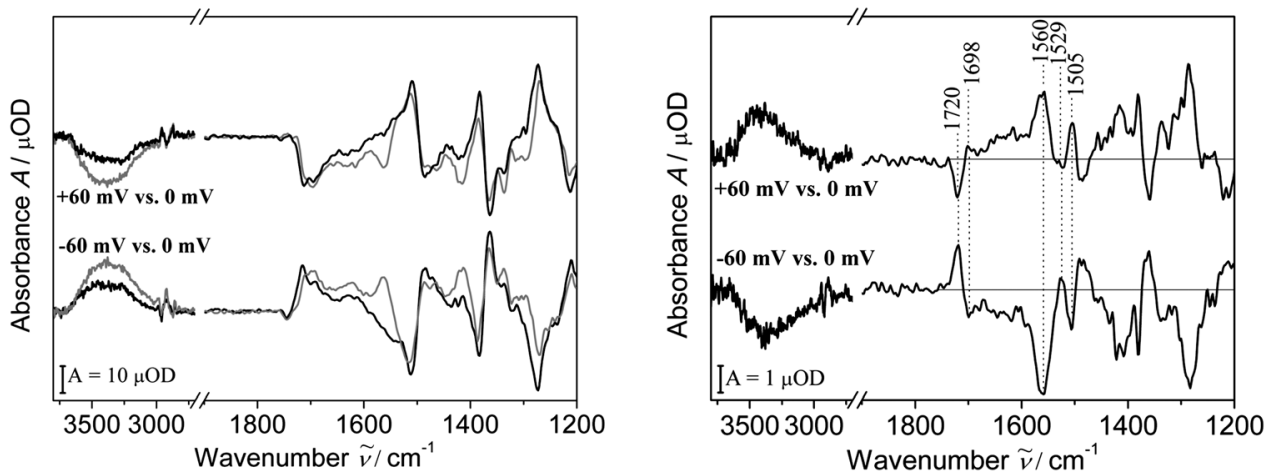

Fig. 4 Left: potential-dependent SEIRA difference spectra at the given potentials $E_{f}\left(v s . E_{O C P}\right.$ ) of hVDAC1-tBLMs (black) and pure tBLMs (grey). Right: potential-dependent SEIRA double-difference spectra calculated as "hVDAC1-tBLM (black) minus tBLM (grey)" at the respective potentials.

(1700-1600 $\left.\mathrm{cm}^{-1}\right)$ shows a broad positive feature attributable to changes in the amide I of the peptide backbone and the glutamine and asparagine side chains as well as to a rearrangement of water molecules. However, due to the low intensity of the amide I of hVDAC1 (vide supra), changes of the protein structure are poorly reflected in this region. The amide II region instead reveals much clearer difference signals with a positive band at $1560 \mathrm{~cm}^{-1}$ and a negative one at $1529 \mathrm{~cm}^{-1}$. Here, we can exclude any contributions from the $\mathrm{CPEO} 3$ modes in this region since its characteristic $\mathrm{C}=\mathrm{O}$ stretching at $1698 \mathrm{~cm}^{-1}$ does not show any notable changes in the presence or absence of hVDAC1, implying that direct (potential-dependent) interactions between hVDAC1 and $\mathrm{CPEO} 3$ molecules can also be ruled out. Thus, the difference band at $1560 \mathrm{~cm}^{-1}$ is attributed exclusively to the amide II stretching of hVDAC1 which was assigned to the $\beta$-sheet connecting turns of the barrel $\left(1558 \mathrm{~cm}^{-1}\right.$; see Fig. 2). The difference band at $1529 \mathrm{~cm}^{-1}$ originates from changes of the $\beta$-sheet structure. Apart from these backbone changes, the positive difference band at $1720 \mathrm{~cm}^{-1}$ and the negative one at $1505 \mathrm{~cm}^{-1}$ might be assigned to amino acid residues located in the turn region and thus close to the Au surface. The band at $c a .1286 \mathrm{~cm}^{-1}$ originates most likely from the $\mathrm{C}-\mathrm{N}$ stretching and $\mathrm{O}-\mathrm{H}$ bending of the monolayer components (vide supra). This band is quite pronounced in the double difference spectrum due to its slight downshift in the hVDAC1-containing tBLMs compared to the pure tBLMs.

The broad amide I region and the amide II band at $1560 \mathrm{~cm}^{-1}$ mainly reflect the movement of the hVDAC1-containing POPC bilayer fragments relative to the Au surface as discussed above (see Fig. 3). The positive difference signals, detected at positive potentials relative to the $E_{\mathrm{OCP}}$, correspond to the movement towards the $\mathrm{Au}$ surface, while negative signals (at negative potentials) correspond to the movement in the opposite direction. However, in contrast to the changes of the $1560 \mathrm{~cm}^{-1}$ band, the amide II difference band at $1529 \mathrm{~cm}^{-1}$, originating from the $\beta$-sheets, displays the opposite sign, i.e. negative sign at positive potentials and vice versa. To interpret the potential-dependent changes of this band, we have to take into account that the transition dipole moment of the amide II band of $\beta$-sheets is oriented in parallel with respect to the propagation direction of the polypeptide chain, ${ }^{20}$ and that the SEIRA surface selection rules lead to a preferential enhancement of modes with a transition dipole moment perpendicular to the Au surface. Thus, $\beta$-sheets oriented in parallel with respect to the surface normal are expected to be SEIRA-active, whereas no enhancement and thus no IR intensity are expected for a perpendicular orientation. Correspondingly, also the characteristic amide I band of the $\alpha$-helix that is oriented parallel to the surface is not enhanced such that possible changes of this structural element remain obscured. Accordingly, there are two possible interpretations of the spectral changes discussed above.

The first model involves a compression and elongation of the entire barrel at positive and negative potentials, respectively, leading to a change in the inclination angle of the $\beta$-sheets (Fig. 5). Consequently, at positive potentials, the $\beta$-sheets are deflected away from the barrel axis resulting in an increased
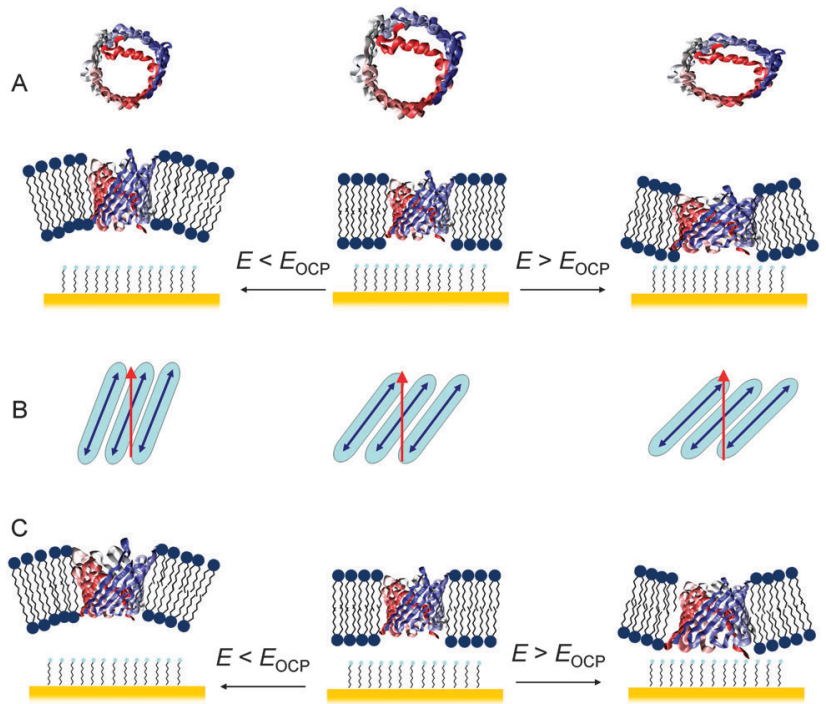

Fig. 5 Proposed structural changes of hVDAC1 upon switching between the open and "closed" states. (A) Compression and elongation of the barrel associated with a change of the pore diameter. (B) Schematic representation of the structural changes associated with an alteration of the inclination angle between the $\beta$-sheets (blue stripes) and the polarized electromagnetic field (red arrow). The blue arrows represent the transition dipole moment changes of the amide II band at $1530 \mathrm{~cm}^{-1}$. (C) Distortion of hVDAC1 leading to a similar inclination of the $\beta$-sheets in the electrode-facing region of the protein associated with the bending of the bilayer membrane. 
angle between the transition dipole moment of the amide II of the $\beta$-sheets and the surface normal. This large-scale protein structural change may account for the negative $1529 \mathrm{~cm}^{-1}$ difference band. At negative potentials, however, the $\beta$-sheets are aligned more parallel towards the barrel axis leading to a positive difference band since the angle between the transition dipole moment of the amide II and the surface normal decreases. These movements of the peptide segments may correspond to a narrowing and widening of the channel pore. Whereas in the case of the elongation the decrease of the pore diameter can be easily understood, the compression is probably associated with a deformation towards an ellipsoidal shape with the $\alpha$-helix acting as a stabilizing element. ${ }^{14}$ Furthermore, the $\beta$-sheets 1 and 19, forming the rather labile parallel pair, may play a crucial role for the oval-shaped "closed" states.

The second model is associated with a bending motion of the POPC bilayer fragments and involves a structural plasticity of the $\beta$-barrel. The bending of the bilayer distorts hVDAC1 into a cone-like structure as shown in Fig. 5. Due to the distancedependent attenuation, SEIRA is most sensitive to changes located close to the Au electrode. Thus, bending of the bilayer towards the surface (at positive potentials) would correspond to an expanded structure of the electrode-facing side of hVDAC1. Effectively, this structure leads to an overall increased inclination angle of the $\beta$-sheets and the surface normal and, thus, to a negative difference band in the SEIRA spectra. On the opposite, bending-up of the bilayer causes the compression of the electrode-facing side of hVDAC1 leading to an increased number of $\beta$-sheet elements with an orientation more parallel to the surface. This orientation change may well account for a positive intensity of the amide II difference band. Such a mechanism may be promoted by the labile 19 stranded $\beta$-sheet motif and the stabilizing $\alpha$-helix in the centre of the pore. In any case, a high flexibility of the bilayer is the basis of such a process.

Even though the $\alpha$-helix is not visible in the SEIRA method, both models derived from the present results are consistent with previous findings on this structural element. ${ }^{18}$ Accordingly, the $\alpha$-helix may not only passively support the global structural changes of the pore. Instead, both the $\beta$-barrel (model 1 ) or the bilayer (model 2) and the $\alpha$-helix may respond to the electric field and contribute to the opening and closing events of the pore in a synergetic manner.

\section{Conclusions}

Based on the concept of tBLMs, model membranes were assembled on nanostructured $\mathrm{Au}$ electrodes that allowed for simultaneous electrochemical (EIS) and spectroscopic (SEIRA) characterization. In this work, we employed this approach for the first time to study the molecular functioning of the large voltage-dependent transmembrane channel protein hVDAC1. EIS and SEIRA spectroscopies were shown to be sensitive tools for monitoring the step-wise assembly of the model membrane, its structural and functional integrity, and the successful incorporation of the protein. In contrast to other types of supported bilayers,
tBLMs offer the advantage of a good control of the transmembrane potential via the electrode potential. This is a prerequisite for switching between the "closed" and open states of the channel protein and analyzing the underlying molecular changes by SEIRA spectroscopy. These changes involve collective motions of the $\beta$-barrel structure that result in a widening or narrowing of the pore. They may be either directly induced by changes in the electric field or are the result of the field-dependent attraction or repulsion of the bilayer with respect to the electrode. The present data are not conclusive on the involvement of the $\alpha$-helix which was previously shown to play a crucial role for the closing and opening mechanism, ${ }^{11}$ since the characteristic amide I mode of this structural element remains invisible in the SEIRA spectrum. This is the drawback of the orientation- and distance-dependence of the IR signal enhancement which is, at the same time, the basis of the high sensitivity and selectivity of this spectroscopic method.

\section{Acknowledgements}

The work was supported by the DFG within the Cluster of Excellence "UniCat" (P.H., J.K.) and the SFB 803 (C.W., C.S.) and the Netherlands Organisation for Scientific Research (NWO) grant 722.011.003 (D.M.).

\section{References}

1 V. Shoshan-Barmatz, V. De Pinto, M. Zweckstetter, Z. Raviv, N. Keinan and N. Arbel, Mol. Aspects Med., 2010, 31, 227-285.

2 T. K. Rostovtseva and M. Colombini, Biophys. J., 1997, 72, 1954-1962.

3 V. E. Kagan, H. A. Bayir, N. A. Belikova, O. Kapralov, Y. Y. Tyurina, V. A. Tyurin, J. Jiang, D. A. Stoyanovsky, P. Wipf and P. M. Kochanek, et al., Free Radicals Biol. Med., 2009, 46, 1439-1453.

4 T. K. Rostovtseva, W. Tan and M. Colombini, J. Bioenerg. Biomembr., 2005, 37, 129-142.

5 R. Benz, Biochim. Biophys. Acta, 1994, 1197, 167-196.

6 M. Colombini, E. Blachly-Dyson and M. Forte, Ion Channels, 1996, 4, 169-202.

7 S. Hiller, J. Abramson, C. Mannella, G. Wagner and K. Zeth, Trends Biochem. Sci., 2010, 35, 514-521.

8 A. G. Komarov, D. Deng, W. J. Craigen and M. Colombini, Biophys. J., 2005, 89, 3950-3959.

9 S. Hiller, R. G. Garces, T. J. Malia, V. Y. Orekhov, M. Colombini and G. Wagner, Science, 2008, 321, 1206-1210.

10 M. Bayrhuber, T. Meins, M. Habeck, S. Becker, K. Giller, S. Villinger, C. Vonrhein, C. Griesinger, M. Zweckstetter and K. Zeth, Proc. Natl. Acad. Sci. U. S. A., 2008, 105, 15370-15375.

11 R. Ujwal, D. Cascio, J.-P. Colletier, S. Faham, J. Zhang, L. Toro, P. Ping and J. Abramson, Proc. Natl. Acad. Sci. U. S. A., 2008, 105, 17742-17747.

12 R. Schneider, M. Etzkorn, K. Giller, V. Daebel, J. Eisfeld, M. Zweckstetter, C. Griesinger, S. Becker and A. Lange, Angew. Chem., Int. Ed., 2010, 49, 1882-1885. 
13 S. Villinger, R. Briones, K. Giller, U. Zachariae, A. Lange, B. L. de Groot, C. Griesinger, S. Becker and M. Zweckstetter, Proc. Natl. Acad. Sci. U. S. A., 2010, 107, 22546-22551.

14 U. Zachariae, R. Schneider, R. Briones, Z. Gattin, J.-P. Demers, K. Giller, E. Maier, M. Zweckstetter, C. Griesinger and S. Becker, et al., Structure, 2012, 20, 1540-1549.

15 M. Colombini, Biochim. Biophys. Acta, 2012, 1818, 1457-1465.

16 V. De Pinto, S. Reina, F. Guarino and A. Messina, J. Bioenerg. Biomembr., 2008, 40, 139-147.

17 C. A. Mannella, J. Struct. Biol., 1998, 121, 207-218.

18 B. Mertins, G. Psakis, W. Grosse, K. C. Back, A. Salisowski, P. Reiss, U. Koert and L.-O. Essen, PLoS One, 2012, 7, e47938.

19 O. Teijido, R. Ujwal, C.-O. Hillerdal, L. Kullman, T. K. Rostovtseva and J. Abramson, J. Biol. Chem., 2012, 287, 11437-11445.

20 A. Barth and C. Zscherp, Q. Rev. Biophys., 2002, 35, 369-430.

21 H. Abrecht, E. Goormaghtigh, J.-M. Ruysschaert and F. Homble, J. Biol. Chem., 2000, 275, 40992-40999.

22 H. Engelhardt, T. Meins, M. Poynor, V. Adams, S. Nussberger, W. Welte and K. Zeth, J. Membr. Biol., 2007, 216, 93-105.

23 A. Deniaud, C. Rossi, A. Berquand, J. Homand, S. Campagna, W. Knoll, C. Brenner and J. Chopineau, Langmuir, 2007, 23, 3898-3905.

24 J. Kozuch, C. Steinem, P. Hildebrandt and D. Millo, Angew. Chem., Int. Ed., 2012, 51, 8114-8117.

25 E. K. Schmitt, M. Nurnabi, R. J. Bushby and C. Steinem, Soft Matter, 2008, 4, 250-253.
26 H. Miyake, S. Ye and M. Osawa, Langmuir, 2002, 4, 973-977. 27 L. J. C. Jeuken, N. N. Daskalakis, X. Han, K. Sheikh, A. Erbe, R. J. Bushby and S. D. Evans, Sens. Actuators, B, 2007, 124, 501-509.

28 J. K. R. Kendall, B. R. G. Johnson, P. H. Symonds, G. Imperato, R. J. Bushby, J. D. Gwyer, C. van Berkel, S. D. Evans and L. J. C. Jeuken, ChemPhysChem, 2010, 11, 2191-2198.

29 F. Kremer and A. Schönhals, Broadband Dielectric Spectroscopy, Springer-Verlag, Berlin, Heidelberg, 2003.

30 E. Goormaghtigh, V. Cabiaux and J.-M. Ruysschaert, Subcell. Biochem., 1994, 23, 405-450.

31 D. Marsh, M. Müller and F. J. Schmitt, Biophys. J., 2000, 78, 2499-2510.

32 A. M. Dwivedi and S. Krimm, J. Phys. Chem., 1984, 88, 620-627.

33 N. Wisitruangsakul, O. Lenz, M. Ludwig, B. Friedrich, F. Lendzian, P. Hildebrandt and I. Zebger, Angew. Chem., Int. Ed., 2009, 48, 611-613.

34 N. Kucerka, S. Tristram-Nagle and J. F. Nagle, J. Membr. Biol., 2005, 208, 193-202.

35 L. J. C. Jeuken, Biophys. J., 2008, 94, 4711-4717.

36 H. H. Mantsch and R. N. McElhaney, Chem. Phys. Lipids, 1991, 57, 213-226.

37 W. Knoll, I. Köper, R. L. C. Naumann and E.-K. Sinner, Electrochim. Acta, 2008, 53, 6680-6689.

38 M. Andersson, H. M. Keizer, C. Zhu, D. Fine, A. Dodabalapur and R. S. Duran, Langmuir, 2007, 23, 2924-2927. 